Amor, M. I. y Serrano Rodríguez, R. (2020). Las competencias profesionales del orientador escolar: el rol que representa desde la visión del alumnado. Revista de Investigación Educativa, 38(1), 71-88.

DOI: http://dx.doi.org/10.6018/rie.321041

\title{
Las competencias profesionales del orientador escolar: el rol que representa desde la visión del alumnado
}

\section{The professional competences of school counselors: the role that they represent from pupils' points of view}

\author{
Dra. María Isabel Amor y Dra. Rocío Serrano Rodríguez \\ Universidad de Córdoba
}

\section{Resumen}

En el presente trabajo se presenta una investigación realizada en los centros de Educación Secundaria Obligatoria (ESO) de Córdoba y su provincia, España, con la finalidad de conocer el rol que desempeña el orientador/a, las funciones y actividades que desarrolla, el tipo de ayuda que le solicita el alumnado cuando acude a él, y cómo son las relaciones que establece con el alumnado y sus familias, desde la visión del alumnado. La muestra estuvo compuesta por 328 alumnos, de los cursos $1^{\underline{o}}, 2^{\circ}, 3^{\circ}$ y $4^{\circ}$ de la ESO, distribuidos en un total de 12 Centros de Educación Infantil y Primaria (CEIP), e Institutos de Educación Secundaria (IES), nueve de ellos de carácter público (76.2\%) y tres de carácter privado (23.8\%). Se utilizó un método de carácter descriptivo y comparativo, para analizar las diferencias entre las puntuaciones medias de los diferentes grupos. Los resultados obtenidos confirman que el alumnado valora positivamente la labor que realiza el orientador/a y las relaciones son muy satisfactorias, aunque existen diferencias en la percepción de su labor en función del tipo de centro y del género. Puede concluirse que la figura del orientador es un factor clave para el éxito académico del alumnado, pero necesita delimitar sus funciones para desempeñar su labor tal y como se determina en la normativa vigente.

Palabras clave: orientador escolar; funciones; rol; educación secundaria.

Correspondencia: Rocío Serrano Rodríguez, rocio.serrano@uco.es. Universidad de Córdoba, Av. San Alberto Magno s/n 14071, Córdoba (España). 


\begin{abstract}
The present research shows a study carried out in Obligatory Secondary Education (OSE) centers in the city and province of Córdoba, Spain. The objective is to discover the role played by school counselors, their duties and the activities they develop. The type of help that the students request from these professionals and theirrelationships with students and families from the learners' perspective is also explored. The sample consisted of 328 students from the $1 s t, 2 n d, 3 r d$ and 4 th years of OSE, distributed throughout 12 Infant and Primary Education Centers (IPEC) and Secondary Education Schools (SES), nine of which were public (76.2\%) and three private $(23.8 \%)$. The method used in order to analyze the differences between the average scores obtained by the different groups was descriptive and comparative. The results support the idea that the students positively value the work done by their counselors, and that their relationships are also very satisfactory. However, the counselors' work is perceived differently depending on the type of center and gender. Finally, it can be concluded that the figure of the counselor is a key factor for the academic success of students, but it is necessary to delimit their duties to work according to the current regulations.
\end{abstract}

Keywords: school counselor; duties; role; secondary education.

\title{
Introducción
}

Hace más de veinte años que en España desde la implantación de la Ley Orgánica 1/1990 de 3 de octubre de Ordenación General del Sistema Educativo (LOGSE), se originó un cambio bastante significativo, no sólo en la organización y estructuración del sistema educativo de forma general, sino que además supuso una mejora en la calidad de la educación resaltando los principios de comprensividad y diversidad, conformes a la realidad social de ese momento. Con esta ley, se consiguió la regulación de la orientación en el sistema educativo y la creación del Departamento de Orientación como órgano de planificación y coordinación de la orientación y la acción tutorial, con la finalidad de desarrollar actuaciones dirigidas principalmente al alumnado de Educación Secundaria.

La LOGSE (1990), establece un modelo organizativo y funcional estructurado en tres niveles de intervención: aula, centro y zona que sustenta el actual modelo de orientación en España. Esta ley, al contrario que las leyes anteriores (Ley Orgánica 5/1980, de 19 de junio, por la que se regula el Estatuto de Centros Escolares, LOECE, 1980, la Ley Orgánica 8/1985, de 3 de julio, reguladora del Derecho a la Educación, LODE, 1985 y la Ley General de Educación de 4 de agosto de 1970, LGE, 1970) incide de manera clara e inequívoca en el impulso de la orientación de forma explícita a largo de su redacción. Actualmente, la Ley Orgánica 8/2013, de 9 de diciembre, para la Mejora de la Calidad Educativa, (LOMCE, 2013) no contempla ninguna atención específica en relación con la orientación, y deja establecido el sistema orientador tal y como hemos citado con anterioridad a partir de la LOGSE y la LOE (Rodríguez, Ocampo, y Sarmiento, 2018).

Podemos afirmar (Amor, 2012) que, la LOGSE no solo consiguió que la orientación se convirtiera en un derecho, sino que, formará parte de las instituciones educativas.

En 2006, se publica la Orden Ministerial del 27 de julio que regula las funciones del Departamento de Orientación con relación al centro, el alumnado, el profesorado y 
las familias. Estas actuaciones del Departamento de Orientación se estructuran en dos pilares: el primero de ellos, dedicado al asesoramiento y la orientación del alumnado, que depende directamente del orientador/a, y el otro, a la atención del alumnado con Necesidades Educativas Especiales (NEE) y grupos específicos, que dependen de otros profesionales que también forman parte del Departamento de Orientación. Entre sus funciones encontramos; participar, animar, asesorar y trabajar conjuntamente en los distintos foros de decisión de la intervención educativa en los centros y en la acción orientadora del alumnado (Motoso, 2002, p.83).

La composición del Departamento de Orientación presenta diferentes singularidades en función del contexto, del centro, de la comunidad e incluso del país en el que nos encontremos. De ahí se deduce que existen diferentes modelos de actuación, estructuras y funciones según la población que deban atender. Según Hervás Avilés (2006, p.77), en España sus funciones están claramente definidas a través de cuatro áreas de intervención:

- Orientación el los procesos de enseñanza y aprendizaje

- Orientación profesional

- Atención a la diversidad

- Orientación para la prevención y el desarrollo.

De acuerdo con Bisquerra (2005), estas áreas no están aisladas, sino que están interrelacionadas y es precisamente su enlace lo que da unidad a la orientación que se dirige al individuo como un todo. El orientador/a debe favorecer la toma de decisiones y las habilidades de autonomía para dotar de herramientas al alumnado ante la incertidumbre que viven y los acontecimientos que se suceden (Villardón y Yáñiz, 2013). Coincidiendo con Velaz de Medrano (2011, p.129): “la orientación en esta etapa debe plantearse como un conjunto de acciones planteadas a lo largo de ella para facilitar y potenciar el aprendizaje, el conocimiento y la gestión por parte del alumnado y de sus propias competencias personales y profesionales".

Martínez-Clares, Pérez y Martínez-Juárez, (2014), insisten en que hay que conceder mayor importancia a la emotividad, a la dimensión relacional, a entender la indecisión y la equivocación como algo propio de la toma de decisiones, promoviendo la reflexión y el autoconocimiento en el alumnado.

Los orientadores/as actuales y futuros, deben tener conocimientos sobre los acontecimientos socioeconómicos, culturales, políticos y laborales "porque para orientar no son suficientes los conocimientos derivados del ámbito psicopedagógico, es necesario también indagar hacia dónde y hacia qué orientamos. Es necesario un análisis de la realidad para comprenderla, interpretarla $y$, si es preciso transformarla, porque forma parte de su actividad profesional" (Santana Vega y Álvarez Pérez, 1996, p.71).

Comprobamos que a menudo, la práctica orientadora manifiesta insuficiencias que obstaculizan conseguir los objetivos que se le atribuyen como acción educativa. Carencias que, según diversos autores (Álvarez González, 2014; McCarthy \& Watson, 2018 y Walker, Shenker \& Hoover, 2010) están relacionadas con el cumplimiento de los principios de prevención, desarrollo e intervención social, con un predominio de la intervención de carácter remedial e individualizada. El escaso protagonismo de 
las funciones de consulta y formación y fundamentalmente, la poca presencia de la orientación en el currículo educativo y en escenarios relacionados con el contexto familiar.

En la línea de lo anterior, la investigación desarrollada por Carey, Martín, Harrington y Michael (2018) sobre las funciones que realiza el orientador/a, concluyen como es necesario realizar una revisión de estas funciones, con la finalidad de aportar claridad a las competencias de estos profesionales.

Por otro lado, Mekgwe y Kok (2017), analizaron las principales consultas que el alumnado realiza a los Departamentos de Orientación en una investigación con más de trescientos alumnos y alumnas en centros educativos de Botswana (Sudáfrica). Concluyeron que, los temas más notables de preocupación entre el alumnado están centrados en la carrera personal, en concreto: la preparación para entrevistas, las carreras disponibles y la búsqueda de empleo. Por otro lado, Welsch y Widen (2019) afirman que, existe una brecha de género en relación con el tipo de consulta que el alumnado realiza al orientador/a. Siendo más notable la presencia de las chicas en estas consultas.

Para Echeverría, (2008, p.38), el desafío está en formar desde un enfoque más global que integre los objetivos que la orientación plantea en la búsqueda del desarrollo integral del alumnado, porque a pesar de todas las debilidades que se le atribuyen al trabajo que desempeñan los orientadores/as en la actualidad (Hernández Rivero y Mederos Santana, 2018; Martínez-Otero, 2018), también existen estudios (Álvarez González, Figuera y Torrado, 2011; Calvo, Haya y Susinos, 2012; Silva Teixeira, Fazendeiro y Braga, 2016) que evidencian la relevancia y la necesidad de su papel en los centros de Educación Secundaria.

Por lo tanto, teniendo en cuenta el recorrido histórico y legislativo por el que han ido desfilando las funciones del orientador/a en España pensamos que es el momento de analizar y reflexionar sobre la realidad educativa en la que nos encontramos, para constatar si estamos haciendo lo que realmente necesita la escuela, el alumnado, la familia y la sociedad en general, y quizás replantearnos las modificaciones necesarias (Martínez-Clarés y Martínez-Juárez, 2011) si fuera necesario.

\section{Método}

\section{Objetivos}

El objetivo general de este estudio, consiste en conocer la labor que desempeña el orientador/a en los centros de Educación Secundaria Obligatoria (ESO) de Córdoba y su provincia, en opinión del alumnado.

Para ello nos planteamos los siguientes objetivos específicos:

- Analizar la percepción que tiene el alumnado sobre el orientador/a de su centro: sus relaciones, las funciones que realiza y la importancia que atribuye a su la labor.

- Comprobar si existen diferencias en las respuestas del alumnado en función de sí conoce o no, al orientador/a de su centro. 
- Comprobar si existen diferencias en las respuestas del alumnado en función del sexo y de la tipología del centro (público o privado) en relación con las dimensiones en las que se divide la escala AFO.

\section{Población y Muestra}

La población objeto de estudio está constituida por el alumnado de Educación Secundaria Obligatoria (ESO) de Córdoba y su provincia. Mediante un muestreo de conveniencia (Otzen \& Manterola, 2017), se seleccionaron 328 alumnos y alumnas de los cursos $1^{\circ}, 2^{\circ}, 3^{\circ}$ y $4^{\circ}$ de la ESO. Distribuidos en un total de 12 Centros de Educación Infantil y Primaria (CEIP), e Institutos de Educación Secundaria (IES) (elegidos por accesibilidad y proximidad) (Tabla 1). Nueve de ellos son de carácter público (76.2\%) y tres de carácter privado (23.8\%). El 48\% son mujeres y el 52\% son hombres, con edades que oscilan entre los 13 y los 17 años $(\mathrm{M}=2.15$; D.T=.844). Se asumió un error muestral del $4 \%$ para un nivel de confianza del $97 \%$.

Tabla 1

Distribución del alumnado participante por cada uno de los centros

\begin{tabular}{|c|c|c|}
\hline Nombre del Centro & $\mathbf{N}$ & $\%$ \\
\hline CEIP Virgen del Carmen & 27 & 8,2 \\
\hline CEIP Antonio Gala & 48 & 14.6 \\
\hline CEIP Francisco de los Ríos & 28 & 8.5 \\
\hline IES La Fuensanta & 26 & 7.9 \\
\hline IES Luis de Góngora & 33 & 10.1 \\
\hline CEIP Luis Carrillo de Sotomayor & 25 & 7.6 \\
\hline CEIP Jesús Nazareno & 22 & 6.7 \\
\hline IES López Neyra & 16 & 4.9 \\
\hline CEIP Márquez de Comares & 26 & 7.9 \\
\hline CEIP San Francisco de Sales & 29 & 8.8 \\
\hline IES Seneca & 30 & 9.1 \\
\hline IES Trassierra & 18 & 5.5 \\
\hline Total & 328 & 100 \\
\hline
\end{tabular}

\section{Instrumento}

Para alcanzar los objetivos previstos, se elaboró un cuestionario con cinco preguntas y un total de 30 ítems, con una escala de valoración tipo Likert con cinco 
niveles de graduación que van desde el menor (1) al mayor grado de acuerdo (5). Los 30 ítems pretenden analizar la figura del orientador/a en los Centros de Educación Secundaria Obligatoria (ESO) y están estructurados en cinco grupos de dimensiones. En el primer grupo (D1), las variables están relacionadas con la percepción que tiene el alumnado sobre el orientador/a de su centro, en la segunda dimensión (D2) se recogieron aquellas variables que están relacionadas con las funciones que desempeña, el tercer grupo de variables (D3) lo conformaron los ítems relacionados con las actividades que habitualmente realiza, la cuarta dimensión (D4) agrupa los ítems relacionados con el tipo de relación que mantiene el orientador/a, con el alumnado y con sus familias, cuando y cómo se produce y grado de satisfacción. Y en el último grupo de variables (D5) se encuentran los ítems relacionados con el tipo de ayuda que solicita el alumnado y las demandas que realiza cuando acude al orientador/a de su centro.

Con el objetivo de garantizar la fiabilidad del instrumento, se realizó una búsqueda documental con el fin de identificar las variables más relevantes objeto de nuestro estudio, así como los instrumentos utilizados (validados y aplicados) en otros estudios similares.

De esta primera fase, se extrajeron un conjunto amplio de preguntas (40). Estas preguntas constituyeron la primera versión del instrumento (Estebaranz, Mingorance y Monescillo, 2001; Jariot, 2010) y fueron sometidas a criterio de expertos, quienes valoraron la relevancia, pertinencia, intensidad, claridad y comprensividad de los ítems (Thomas y Nelson, 2007). Se les solicitó que respondieran con valores entre; $1=$ en desacuerdo con el ítem, hasta $5=$ totalmente de acuerdo con el ítem. Se estableció como criterio eliminar todas aquellas preguntas que fueran valoradas con 1, 2, 3 y 4 y se mantuvieron las que había un acuerdo del 95\% entre los expertos. Tras la información obtenida sobre la idoneidad de cada uno de ellos, se eliminaron los que no se consideraron adecuados al objeto del estudio, y se obtuvo otra versión de la escala con 35 ítems. Con este procedimiento se cumplió la validez de contenido, al realizar una previa revisión exhaustiva de investigaciones cuyo objetivo e instrumentos aplicados fueron similares a nuestro objeto de estudio, así como la prueba de jueces realizada con el conjunto inicial de ítems del instrumento.

Para comprobar la validez de comprensión del cuestionario, se realizó un estudio piloto a una sub-muestra de 20 alumnos y alumnas de forma presencial (Alaminos y Castejón, 2006). Se analizó el grado de comprensión desde un punto de vista cualitativo; registrándose las preguntas, dudas y sugerencias que los sujetos realizaron en la sesión presencial de cumplimentación. Los resultados obtenidos nos aportaron información sobre la eliminación de aquellos ítems que no respondían adecuadamente (5).

Posteriormente, se calculó la fiabilidad mediante el coeficiente alfa de Cronbach, obteniendo un $\alpha=.85$ para los 30 ítems. En este caso la eliminación de alguno de los ítems de la escala no suponía ningún incremento significativo en la fiabilidad, por lo que se decidió mantenerlos.

Finalmente, con la escala para el "Análisis de la Figura del Orientador en los centros (AFO)" definitiva se procedió a su aplicación. En la tabla siguiente (Tabla 2) se relacionan los ítems de la escala final y su distribución por dimensiones. 
Tabla 2

Relación de los Ítems de la escala AFO y su distribución por dimensiones

\begin{tabular}{|c|c|c|}
\hline Dimensiones & Variables & Ítems \\
\hline \multirow{7}{*}{ D1 } & V.1 & $\begin{array}{l}\text { Piensas que es importante la figura del orientador en tu cen- } \\
\text { tro }\end{array}$ \\
\hline & V.2 & Crees que la labor que realiza en tu centro es necesaria \\
\hline & V.3 & Solicitarías ayuda al orientador ante un problema personal \\
\hline & V.4 & Crees que te puede ayudar en tus estudios \\
\hline & V.5 & Consideras que las actividades que plantea son interesantes \\
\hline & V.6 & $\begin{array}{l}\text { Crees que puede ayudarte para tomar decisiones sobre tu } \\
\text { futuro académico }\end{array}$ \\
\hline & V.7 & Cuando has solicitado ayuda o consulta te ha atendido \\
\hline \multirow{6}{*}{ D2 } & V.8 & Crees que orienta e informa sobre aspectos académicos \\
\hline & V.9 & Crees que orienta e informa sobre aspectos profesionales \\
\hline & V.10 & Crees que orienta y asesora sobre aspectos personales \\
\hline & V.11 & Crees que puede ayudar en tu aprendizaje \\
\hline & V.12 & $\begin{array}{l}\text { Crees que puede orientar y prevenir las dificultades de } \\
\text { aprendizaje }\end{array}$ \\
\hline & V.13 & $\begin{array}{l}\text { Crees que informa y asesora a tus padres sobre tu desarrollo } \\
\text { académico }\end{array}$ \\
\hline \multirow{5}{*}{ D3 } & V.14 & $\begin{array}{l}\text { Desarrolla actividades para prevenir el fracaso en tus estu- } \\
\text { dios }\end{array}$ \\
\hline & V.15 & $\begin{array}{l}\text { Realiza sesiones informativas sobre los posibles itinerarios } \\
\text { académicos }\end{array}$ \\
\hline & V.16 & $\begin{array}{l}\text { Desarrolla actividades para aumentar vuestra motivación } \\
\text { hacia los estudios }\end{array}$ \\
\hline & V.17 & $\begin{array}{l}\text { Realiza actividades para que conozcáis vuestras aptitudes } \\
\text { y habilidades }\end{array}$ \\
\hline & V.18 & $\begin{array}{l}\text { Desarrolla actividades para fomentar la interacción en el } \\
\text { grupo }\end{array}$ \\
\hline \multirow{6}{*}{ D4 } & V.19 & Es individual y personalizada \\
\hline & V.20 & Aporta información y asesoramiento \\
\hline & V.21 & Es muy accesible y facilita el contacto \\
\hline & V.22 & Sólo se establece cuando existe una necesidad \\
\hline & V.23 & La relación con el alumnado es buena y satisfactoria \\
\hline & V.24 & La relación con la familia es buena y satisfactoria \\
\hline
\end{tabular}




\begin{tabular}{ccl}
\hline Dimensiones & Variables & \multicolumn{1}{c}{ Ítems } \\
\hline V.25 & $\begin{array}{l}\text { Has solicitado ayuda en el estudio, superación de asigna- } \\
\text { turas y realización de trabajos. } \\
\text { V.26 }\end{array}$ & $\begin{array}{l}\text { Has solicitado ayuda para conocer tus características, apti- } \\
\text { tudes e intereses } \\
\text { V.27 }\end{array}$ \\
V.28 & $\begin{array}{l}\text { Has solicitado información sobre estudios, becas, cursos, } \\
\text { actividades. } \\
\text { Has solicitado ayuda para tomar decisiones sobre tus es- } \\
\text { tudios } \\
\text { V.29 }\end{array}$ & $\begin{array}{l}\text { Has solicitado ayuda para relacionarte y llevarte bien con } \\
\text { tus compañeros y tus profesores }\end{array}$ \\
V.30 & Has solicitado información sobre técnicas de estudio \\
\hline
\end{tabular}

\section{Procedimiento de recogida y análisis de datos}

La escala AFO fue administrada durante los meses de marzo y abril del curso académico 2014/15. Se especificó el objeto de estudio de la investigación, su carácter voluntario, así como el compromiso de confidencialidad de los datos obtenidos. El procedimiento se realizó en horas lectivas del curso, con previo acuerdo de la dirección del centro, así como del profesorado, que impartía clase en ese momento.

Con la intención de dar respuesta al primero de los objetivos se realizó un análisis descriptivo de los valores medios y las desviaciones típicas, así como la frecuencia de las respuestas a cada uno de los ítems.

Para el segundo objetivo, se utilizó la prueba estadística $t$ de Student. Era necesario comprobar la existencia de diferencias en las respuestas entre el alumnado que indican conocer al orientador/a de su centro $(\mathrm{N}=1)$ y alumnado que indican no conocer al orientador/a de su centro $(\mathrm{N}=2)$.

Para el último de los objetivos, aplicamos de nuevo la prueba $t$ de Students con la intención de comprobar si existen diferencias en función de la variable sexo y el carácter o tipología de centro educativo (público o privado), con relación a las dimensiones de la escala AFO: D1, Percepción; D2, Funciones; D3, Actividades; D4, Relaciones y D5, Demandas.

Se utilizó la prueba de Levene como criterio para asumir si las varianzas eran iguales o no. El nivel de confianza ha sido del 95\% ( $\mathrm{p}>.05)$.

Para los análisis descritos se utilizó el programa SPSS.22.

\section{Resultados}

A través de los análisis descriptivos los resultados obtenidos demuestran que los estudiantes atribuyen una importancia alta a las variables relacionadas con la orientación para prevenir las dificultades en los estudios ítem 12 (V.12=4.08\%), seguida de la 
orientación sobre aspectos académicos ítem 8 (V.8=4.05\%). Por otro lado, el alumnado percibe como menos importante recibir asesoramiento sobre técnicas de estudio ítem $30(\mathrm{~V} .30=2.92 \%)$, y orientación para conocerse a sí mismo ítem 26 (V.26=2.64\%). En un segundo nivel de importancia, se localizan conceptos como: recibir información sobre estudios posteriores, becas, cursos, etc., con una media de $2.60 \%$, ítem 27 (V.27), seguida de la ayuda para tomar decisiones sobre su futuro académico ítem 6 (V.6=2.59\%) y, por último recibir ayuda para relacionarse con sus compañeros/as y con sus profesores/ as ítem 29 (V.29=2.52\%).

Comparando los valores de las medias en función de si el alumnado conoce o no al orientador/a, podemos observar (Tabla 3), que en la mayoría de las medias, los valores más altos se encuentran en el grupo de alumnos que sí conocen al orientador/a (N1), puntuando más alto en los siguientes ítems: en primer lugar en el ítem 1 (V.1) que describe la importancia que se le atribuye a esta figura $(\mathrm{M}=3.73, \mathrm{D} . \mathrm{T} .=1.070)$, seguido del ítem 2 (V.2) que destaca la labor que realiza (M=3.94, D.T.=.962) y el ítem 4 (V.4) la ayuda que le puede prestar en sus estudios $(\mathrm{M}=3.38$, D.T.=1.124). Finalmente en el ítem 5 (V.5), el alumnado matiza la gran relevancia en las actividades que realiza dirigidas al alumnado (M=3.38, D.T.=1.027).

Sin embargo, los ítems cuyos valores puntúan a favor del grupo N2 (alumnado que no conoce al orientador/a de su centro), son los siguientes: el ítem 3 (V.3) en el que el alumnado opina que no acudiría al orientador/a para solicitar ayuda relacionada con aspectos personales (M=3.25, D.T.=1.412), y en el ítem 10 (V.10) en el que estima que el orientador/a no orienta y asesora en el ámbito personal $(\mathrm{M}=3.68, \mathrm{D} . \mathrm{T}=1.301)$. Por último, en el ítem 7 (V.7) el alumnado considera que no siempre obtiene respuesta cuando solicita la ayuda del orientador/a en su centro (M=4.00, D.T.=.894).

Tabla 3

Estadísticos descriptivos de la escala AFO atendiendo a la variable si conoce al orientador/a de su centro

\begin{tabular}{lcccc}
\hline Variables & Conoce al orientador/a & $\mathbf{N}$ & $\mathbf{M}$ & D.T. \\
\hline \multirow{2}{*}{ V.1 } & Sí & 219 & 3.73 & 1.070 \\
& No & 24 & 3.29 & 1.429 \\
\hline \multirow{2}{*}{ V.2 } & Sí & 273 & 3.94 & .962 \\
& No & 49 & 3.65 & 1.200 \\
\hline \multirow{2}{*}{ V.3 } & Sí & 268 & 3.22 & 1.463 \\
& No & 51 & 3.25 & 1.412 \\
\hline \multirow{2}{*}{ V.4 } & Sí & 262 & 3.38 & 1.124 \\
& No & 47 & 2.81 & 1.313 \\
\hline \multirow{2}{*}{ V.5 } & Sí & 265 & 3.38 & 1.027 \\
& No & 46 & 3.26 & .953 \\
\hline \multirow{2}{*}{ V.6 } & Sí & 269 & 3.70 & 1.110 \\
& No & 49 & 3.20 & .979 \\
\hline
\end{tabular}




\begin{tabular}{|c|c|c|c|c|}
\hline Variables & Conoce al orientador/a & $\mathbf{N}$ & $\mathbf{M}$ & D.T. \\
\hline \multirow{2}{*}{ V.7 } & Sí & 220 & 3.82 & 1.287 \\
\hline & No & 36 & 4.00 & .894 \\
\hline \multirow{2}{*}{ V.8 } & Sí & 250 & 4.07 & 1.033 \\
\hline & No & 46 & 3.93 & 1.323 \\
\hline \multirow{2}{*}{ V.9 } & Sí & 268 & 3.98 & 1.022 \\
\hline & No & 51 & 3.88 & 1.211 \\
\hline \multirow{2}{*}{ V.10 } & Sí & 261 & 3.57 & 1.140 \\
\hline & No & 50 & 3.68 & 1.301 \\
\hline \multirow{2}{*}{ V.11 } & Sí & 271 & 3.89 & 1.000 \\
\hline & No & 52 & 3.60 & 1.317 \\
\hline \multirow{2}{*}{ V.12 } & Sí & 268 & 4.07 & .935 \\
\hline & No & 50 & 4.14 & 1.010 \\
\hline \multirow{2}{*}{ V.13 } & Sí & 271 & 3.48 & 1.157 \\
\hline & No & 52 & 3.54 & 1.290 \\
\hline \multirow{2}{*}{ V.14 } & Sí & 257 & 3.42 & 1.267 \\
\hline & No & 47 & 3.06 & 1.309 \\
\hline \multirow{2}{*}{ V.15 } & Sí & 246 & 3.54 & 1.207 \\
\hline & No & 44 & 3.32 & 1.475 \\
\hline \multirow{2}{*}{ V.16 } & Sí & 243 & 3.44 & 1.128 \\
\hline & No & 44 & 3.20 & 1.407 \\
\hline \multirow{2}{*}{ V.17 } & Sí & 237 & 3.31 & 1.162 \\
\hline & No & 46 & 2.98 & 1.291 \\
\hline \multirow{2}{*}{ V.18 } & Sí & 240 & 3.38 & 1.200 \\
\hline & No & 43 & 3.07 & 1.387 \\
\hline \multirow{2}{*}{ V.19 } & Sí & 264 & 3.61 & 1.235 \\
\hline & No & 48 & 3.04 & 1.368 \\
\hline \multirow{2}{*}{ V.20 } & Sí & 268 & 3.85 & 1.040 \\
\hline & No & 51 & 3.76 & 1.142 \\
\hline \multirow{2}{*}{ V.21 } & Sí & 263 & 3.52 & 1.105 \\
\hline & No & 50 & 3.74 & 1.157 \\
\hline
\end{tabular}




\begin{tabular}{|c|c|c|c|c|}
\hline Variables & Conoce al orientador/a & $\mathbf{N}$ & $\mathbf{M}$ & D.T. \\
\hline \multirow{2}{*}{ V.22 } & Sí & 264 & 3.38 & 1.208 \\
\hline & No & 50 & 3.50 & 1.147 \\
\hline \multirow{2}{*}{ V.23 } & Sí & 263 & 3.76 & 1.060 \\
\hline & No & 48 & 3.40 & 1.180 \\
\hline \multirow{2}{*}{ V.24 } & Sí & 264 & 3.74 & 1.025 \\
\hline & No & 48 & 3.52 & 1.203 \\
\hline \multirow{2}{*}{ V.25 } & Sí & 266 & 2.97 & 1.471 \\
\hline & No & 50 & 3.44 & 1.358 \\
\hline \multirow{2}{*}{ V.26 } & Sí & 263 & 2.62 & 1.387 \\
\hline & No & 49 & 2.78 & 1.571 \\
\hline \multirow{2}{*}{ V.27 } & Sí & 263 & 2.57 & 1.476 \\
\hline & No & 46 & 2.78 & 1.534 \\
\hline \multirow{2}{*}{ V.28 } & Sí & 257 & 2.53 & 1.389 \\
\hline & No & 48 & 2.92 & 1.596 \\
\hline \multirow{2}{*}{ V.29 } & Sí & 260 & 2.50 & 1.340 \\
\hline & No & 49 & 2.63 & 1.510 \\
\hline \multirow{2}{*}{ V.30 } & Sí & 272 & 2.94 & 1.494 \\
\hline & No & 50 & 2.80 & 1.525 \\
\hline
\end{tabular}

La prueba $t$ para muestras independientes, detectó diferencias significativas a favor del alumnado que sí conocía al orientador/a de su centro (N1), en el ítem (V.6) "Crees que puede ayudarte para tomar decisiones sobre tu futuro académico" $\left(t_{318}=2.942\right.$, $\mathrm{p}<.05)$. Igualmente, existen diferencias significativas entre las dos muestras, a favor del alumnado que sí conoce al orientador/a (N1), cuando se le pregunta al alumnado sobre la relación que mantiene el orientador/a, concretamente en el ítem 19 (V.19) en referencia a la relación del orientador/a con el alumnado y con sus familias, "Es individual y personalizada" $\left(\mathrm{t}_{312}=2.884, \mathrm{p}<.05\right)$, y en el ítem (V.23) "La relación con el alumnado es buena y satisfactoria" $\left(\mathrm{t}_{311}=2.130, \mathrm{p}<.05\right)$.

En cambio, cuando se le pregunta al alumnado sobre el tipo de ayuda o las demandas que solicita cuando acude al orientador/a de su centro, encontramos diferencias significativas a favor del alumnado que no conoce al orientador/a de su centro (N2), en las respuestas al ítem "Has solicitado ayuda en el estudio, superación de asignaturas y realización de trabajos" (V.25), con los siguientes resultados entre los dos grupos $\left(\mathrm{t}_{316}=-2.097, \mathrm{p}<.05\right)$. 
Tabla 4

Estadísticos descriptivos de las dimensiones de la escala AFO según la variable sexo

\begin{tabular}{lcccc}
\hline Dimensiones & Sexo & N & M & D.T. \\
\hline \multirow{2}{*}{ (D1) Percepción } & M & 157 & 3.29 & .794 \\
& $\mathrm{H}$ & 171 & 3.13 & .823 \\
(D2) Funciones & $\mathrm{M}$ & 157 & 3.81 & .661 \\
& $\mathrm{H}$ & 171 & 3.56 & .824 \\
(D3) Actividades & $\mathrm{M}$ & 157 & 3.07 & 1.096 \\
& $\mathrm{H}$ & 171 & 2.89 & 1.166 \\
(D4) Relaciones & $\mathrm{M}$ & 157 & 3.54 & .676 \\
& $\mathrm{H}$ & 171 & 3.38 & .789 \\
(D5) Demandas & $\mathrm{M}$ & 157 & 2.55 & 1.051 \\
& $\mathrm{H}$ & 171 & 2.62 & 1.058 \\
\hline
\end{tabular}

Atendiendo a la diferencia de sexo entre los participantes, podemos observar (Tabla 4) que, en la mayoría de ellas, las mujeres presentan valores más altos en las puntuaciones de las medias. La prueba $t$ de Students para muestras independientes detectó diferencias significativas a favor de las mujeres en las dimensiones: (D2) $\left(\mathrm{t}_{328}=3.018\right.$, $\mathrm{p}<.05)$ relacionada con las funciones que desempeña el orientador/a en el centro, y en la dimensión (D4) que agrupa las variables relacionadas con el tipo de relación que establece el orientador/a con el alumnado y con sus familias $\left(\mathrm{t}_{328}=2.035, \mathrm{p}<.05\right)$.

Tabla 5

Estadísticos descriptivos de las dimensiones de la escala AFO según el tipo de centro

\begin{tabular}{ccccc}
\hline Dimensiones & Tipo de Centro & N & M & D.T. \\
\hline \multirow{2}{*}{ (D1) Percepción } & Público & 250 & 3.17 & .808 \\
& Privado & 78 & 3.35 & .814 \\
& Público & 250 & 3.63 & .794 \\
(D2) Funciones & Privado & 78 & 3.82 & .623 \\
& Público & 250 & 2.90 & 1.146 \\
(D3) Actividades & Privado & 78 & 3.22 & 1.070 \\
& Público & 250 & 3.45 & .761 \\
(D4) Relaciones & Privado & 78 & 3.49 & .676 \\
& Público & 250 & 2.63 & 1.079 \\
\hline
\end{tabular}


En relación con el tipo de centro encontramos que, en la mayoría de las variables se obtienen medias de rango más elevadas en las respuestas del alumnado de centros privados, a excepción de la (D5) en la que la media puntúa mas alta (2.63\%) a favor de los centros públicos (Tabla 5). En esta dimensión, se centran los ítems relacionados con las demandas que realiza el alumnado cuando acude al orientador/a de su centro.

La prueba $t$ de Students para muestras independientes, detectó diferencias significativas a favor de los centros privados en la dimensión (D1), que agrupa los ítems relacionados con la percepción que tiene el alumnado sobre la figura del orientador/a en el centro $\left(t_{328}=-1.782, \mathrm{p}<.05\right)$, y en la (D3), que agrupa los ítems relacionados con las actividades que desarrolla el orientador/a en el centro $\left(t_{328}=-2.154, \mathrm{p}<.05\right)$.

\section{Discusión y conclusiones}

En este estudio, se ha observado que la valoración del alumnado en relación a la figura del orientador/a, en general, es bastante positiva. Principalmente, la importancia de su labor la sitúan en la ayuda que les puede aportar en aspectos relacionados con la orientación académica, las dificultades que puedan tener en sus estudios, la evaluación y las dificultades de aprendizaje. Resultados en consonancia con las aportaciones de Mekgwe y Kok (2017).

El alumnado considera el rol del orientador/a como un factor clave para atender a las necesidades educativas del estudiante (Boza, 2005; Ferrandis, Grau y Fortes, 2010). Se trata de optimizar sus capacidades y capacitarle para afrontar las dificultades que puedan aparecer a lo largo de su itinerario académico (Amor, 2016).

A la orientación personal del alumnado, relacionada con la ayuda y el asesoramiento necesario para afrontar y mejorar las relaciones con el resto de sus compañeros y con sus profesores/as (Bisquerra, 2005, 2013), el alumnado atribuye menos importancia, quizás porque en nuestro país el modelo de orientación más utilizado hasta el momento (Martínez-Clarés y Martínez-Juárez, 2011) ha sido el académico y obliga a que la orientación personal pase a un segundo plano. Algunos autores, identifican este proceso de orientación con el Modelo de Orientación alemán: centrado en la formación académica, como complemento a los contenidos de la asignatura (Gairín, Armengol, Muñoz y Rodríguez-Gómez, 2015).

Sin embargo, el Modelo de Orientación anglosajón centrado en el desarrollo personal e interpersonal del alumnado, según Bisquerra, (2013), incluso siendo el más promovido por el marco europeo de Educación Superior, (Gairín, et. al., 2015) es el que menos se pone en práctica en todas las etapas del sistema educativo español.

Numerosos estudios confirman, que desde la dimensión personal podemos desarrollar competencias relacionadas con el saber, el hacer y el ser en los estudiantes (Torrecilla, Rodríguez, Herrera y Martín, 2013). Actualmente, se promueve un Modelo de Orientación basado en el desarrollo completo del alumnado, (Amor y Dios, 2017 y Velaz de Medrano, González Benito y López Martín, 2018) como respuesta a las necesidades que se plantean en la praxis diaria de los centros educativos. Un Modelo Integral que aborde aspectos académicos, personales, profesionales y sociales en la formación de los estudiantes puede contribuir, no solo a una formación armónica y completa del alumnado, sino también a incrementar la calidad en la educación (Amor, 2016). 
El alumnado también considera menos significativo recibir formación sobre técnicas de estudio cuando acude para solicitar ayuda al orientador/a. Estudios anteriores (Mendieta Toledo, L.B.; Mendieta Toledo, L.R. y Chamba Zambrano, 2015) evidencian la importancia de la aplicación de técnicas de estudio, porque produce efectos positivos en el rendimiento escolar de los estudiantes.

Según una investigación realizada por Muelas y Beltrán (2011), en el alumnado de la ESO, las técnicas de estudio más apropiadas para esta etapa son la lectura, la toma y elaboración de apuntes, las anotaciones marginales, el subrayado, el resumen, el esquema, los mapas conceptuales, la memorización y el recuerdo, los recursos nemotécnicos, los mapas mentales, entre otras. (p.180).

Con los resultados obtenidos en este trabajo, también hemos podido comprobar que el alumnado que conoce al orientador/a, y por lo tanto ha acudido para recibir su apoyo en algún momento, es el que manifiesta que le puede proporcionar ayuda para tomar decisiones y configurar su itinerario académico (Velaz de Medrano, González Benito y López Martín, 2018). Además considera que la relación que existe entre ambos es muy buena y satisfactoria, a la vez que bastante personalizada.

Del mismo modo, hemos comprobado que el grupo de alumnos/as que afirma no conocer al orientador/a ratifica, que no acude para solicitar ayuda a esta figura, ni siquiera para el asesoramiento en aspectos académicos relacionados con las asignaturas, los contenidos o los trabajos que debe realizar. Este planteamiento nos hace reflexionar sobre la posición que adopta el orientador/a actualmente, un tanto alejada de las funciones o el liderazgo, que por su papel relevante en los procesos de cambio y mejora escolar debería tener (Janson, Stone \& Clark, 2009).

Existen centros en los que al orientador/a acuden muy pocos alumnos/as, incluso en muchas ocasiones, es conocido por un escaso número (Cano, y García Nieto, 2009). Quizás por la concepción, aún persistente, del orientador/a como psicólogo escolar que sólo ejerce labores de ajuste personal y emocional. Además, uno de los inconvenientes que tiene el rol del orientador/a es la gran cantidad de funciones y demandas, algunas de ellas, que van más allá de las establecidas legislativamente (Hernando y Montilla, 2009), y por tanto conlleva a una confusión por parte, no solo de ellos, sino de toda la comunidad educativa de la que forman parte. Como nos anticipaban Carey, Martín, Harrington y Michael (2018), sería necesario hacer una revisión de las funciones y competencias de estos profesionales.

La realidad nos demuestra que haber involucrado a los orientadores en actividades poco relacionadas con la orientación y el asesoramiento al alumnado (Velaz de Medrano, González Benito y López Martín, 2018) ha dado lugar a la existencia de barreras por parte del alumnado y a la falta de claridad en sus funciones, lo cual les impide desarrollarse y dedicarse de una forma más directa a las tareas propias que su rol determina (Lambie \& Williamson, 2004, p.124).

En relación con las diferencias encontradas en función del sexo, hemos observado que en general son las mujeres las que puntúan más alto en todas las dimensiones, pero de manera más significativa, en primer lugar, en las variables relacionadas con las funciones que desempeña el orientador/a, en concreto acciones dirigidas a la orientación de aspectos académicos, personales y la prevención de dificultades académicas, en segundo lugar y coincidiendo con investigaciones anteriores (Hernández y Mederos, 
2018; Martínez-Otero, 2018; Welsch y Widen, 2019) en las variables relacionadas con el tipo y la calidad en las relaciones que establece el orientador/a, con ellas y con su familia.

En el análisis de los resultados en función del tipo de centro educativo, público o privado, es el alumnado de los centros privados los que apuntan hacia valores más altos en la mayoría de las variables, aunque se detectan algunas diferencias. Para conocer de forma más exacta donde se encuentran estas diferencias, comprobamos que el alumnado de los centros privados tiene una mejor percepción del orientador/a de su centro, y valora de forma positiva las actividades que realiza. En estudios anteriores (Bolu \& Oredugba, 2017 y Cano y García Nieto, 2009), también se encontró una mejor percepción de las funciones que del orientador/a en las respuestas del alumnado de centros privados. No obstante, los alumnos/as de los centros públicos valoran más positivamente la ayuda y demanda que solicitan al orientador/a, en concreto, en aspectos relacionados con la orientación para el estudio, la superación de asignaturas y la realización de trabajos, además de la ayuda para tomar decisiones sobre sus estudios.

Nos gustaría resaltar que la importancia y la necesidad de la figura del orientador/a en la Enseñanza Secundaria en España está ampliamente demostrada, en este trabajo y en muchos más en nuestro país (Álvarez González, 2014; Bisquerra, 2013; Calvo, Haya y Susinos, 2012; Martínez-Clares y Martínez-Juárez, 2011; Muñoz y Gairín, 2013) y más allá del ámbito nacional (López Martín y González Villanueva, 2018 y McCarthy \& Watson 2018 y Walker, Shenker \& Hoover, 2010). El problema posiblemente radique en definir sus funciones y priorizar sus actuaciones en aquellos ámbitos y espacios, para los que fue creada esta figura. La realidad con la que nos encontramos actualmente en la práctica aún se encuentra muy alejada del modelo teórico en el que se basa la figura del orientador/a (Velaz de Medrano et al., 2001).

Como posible limitación resaltamos el sesgo que conlleva obtener datos y conclusiones a partir de las propias percepciones y opiniones únicamente desde la visión el alumnado. Una triangulación de la información desde la perspectiva del alumnado, el profesorado y los orientadores enriquecería los resultados de investigación.

Para futuras investigaciones se sugiere ampliar la muestra y el tipo de instrumentos para la recogida de información, tales como la observación, las entrevistas, etc., Esta triangulación de la información permitiría conocer con mayor detalle y en profundidad la realidad del trabajo del orientador/a en los centros. Igualmente, se propone estudiar otro tipo de variables como, la experiencia y la formación del orientador/a.

\section{Referencias}

Alaminos, A. y Castejón, J. L. (2006). Elaboración, análisis e interpretación de encuestas, cuestionarios y escalas de opinión. Alcoy: Marfil.

Álvarez González, M. (2014). La orientación y la tutoría universitaria en la formación de los grados y posgrados. Las transiciones académicas. En Figuera, P. (Ed.). Persistir con éxito en la universidad: de la investigación a la acción. (pp. 175-203). Barcelona: Laertes.

Álvarez González, M., Figuera, P. y Torrado, M. (2011). La problemática de la transición Bachillerato-Universidad en la Universidad de Barcelona. Revista Española de Orientación y Psicopedagogía, 22(1), 15-27. Doi: 10.5944/reop.vol.22.num.1.2011.74 
Amor, M.I. (2015). La Orientación y la Tutoría Universitaria como elementos para la calidad y la innovación en la Educación Superior. Modelo de Acción Tutorial. Revista de Teoría de la Educación, 27, 247-253.

Amor, M.I. (2016). Evaluación de la orientación y la tutoría en la Facultad de Ciencias de la Educación de la Universidad de Córdoba. Educatio Siglo XXI, 34(1), 93-112.

Amor, M.I. y Dios, I. (2017). La Tutoría Universitaria: Un espacio para la Orientación Personal, Académica y Profesional en la Formación Inicial del Profesorado. Revista Española de Orientación y Psicopedagogía (REOP), 28(1), 119-130.

Bisquerra, R. (2005). Marco conceptual de la orientación psicopedagógica. Revista Mexicana de Orientación Educativa. http://www.remo.ws/revistas/remo-6.pdf

Bisquerra, R. (2013). Orientación, tutoría y educación emocional. Madrid: Síntesis.

Bolu, S. y Oredugba, O. (2017). Influence of couselling services on perceived academic performance of secundary school students in Lagos State. International Journal of Instruction, 10(2), 211-228.

Boza, A. (2005). La orientación en educación secundaria: una perspectiva cualitativa desde los orientadores Revista Española de Orientación y Psicopedagogía, 16(1), 129-146. Doi: https://doi.org/10.5944/reop.vol.16.num.1.2005.11366

Calvo, A., Haya, I. y Susinos, T. (2012). El rol del orientador en la mejora escolar. Una investigación centrada en la voz del alumnado como elemento de cambio. Revista de Investigación en Educación, 10 (2), 7-20. Recuperado de http://webs.uvigo.es/reined/

Cano, J. y García Nieto, N. (2009). Percepción del orientador acerca de la importancia que atribuye la comunidad educativa a sus funciones. Contextos Educativos, 12, 57-75.

Carey, C., Martin, I., Harrington, K. y Trevisan, M. (2018). Competence in Program Evaluation and Research Assessed by State School Counselor Licensure Examinations. Professional School Couseling, 22(1), 1-11.

Echeverría, B. (2008). Configuración de la profesionalidad. En B. Echeverría (Coord.), Orientación Profesional (pp. 23-68). Barcelona: UOC.

Estebaranz, A., Mingorance, P. y Monescillo, M. (2001). La construcción de la función orientadora en los centros de Educación Secundaria. Una visión de sus protagonistas. Fuentes, 3, 9-94.

Ferrandis, M. V., Grau, C. y Fortes, M. C. (2010). El profesorado y la Atención a la diversidad en la ESO. Revista Educación Inclusiva, 3(2), 11-28.

Gairín, J., Armengol, C., Muñoz J. L. y Rodríguez-Gómez, D. (2015). Orientación y tutoría en las prácticas profesionalizadoras. Propuesta de estándares de calidad. Revista Española de Orientación y Psicopedagogía, 26(2), 26-41.

Hernández Rivero, V. M. y Mederos Santana, Y. (2018). Papel del orientador/a educativo como asesor/a: funciones y estrategias de apoyo. Revista Española de Orientación y Psicopedagogía, 29(1), 40-57. Doi: https://doi.org/10.5944/reop.vol.29.num.1.2018.23293

Hernando, A. y Montilla, M. V. (2009). El estudio de los roles y funciones de los orientadores de secundaria utilizando la técnica de los grupos de discusión. Revista Española de Orientación y Psicopedagogía, 20(1), 29-39.

Hervás Avilés, M. R. (2006). Orientación e intervención psicopedagógica y procesos de cambio. Granada: Grupo Editorial Universitario.

Janson, C., Stone, C. \& Clark, M. A. (2009). Stretching Leadership: a Distributed Perspective for School Counselor Leaders. Professional School Counseling, 13(2), 98-106. 
Jariot, M. (2010). Cómo implicar al equipo docente en el proceso de orientación. Un ejemplo de superación del enfoque de servicios. Revista Española de Orientación y Psicopedagogía, 21(1), 45-58.

Lambie, G. y Williamson, L. (2004). The Challenge to Change from Guidance Counseling to Professional School Counseling: A Historical Proposition. Professional School Counseling, 8(2), 124-131.

Ley Orgánica 8/2013, de 9 de diciembre, para la Mejora de la Calidad Educativa. (Boletín Oficial del Estado no 295 de 10 de diciembre de 2013). https://www.boe.es/ eli/es/lo/2013/12/09/8/con

Ley Orgánica 1/1990, de 3 de octubre, de Ordenación General del Sistema Educativo (Boletín Oficial del Estado no 238 de 4 de octubre de 1990). https://www.boe.es/boe/ dias/1990/10/04/pdfs/A28927-28942.pdf.

Ley Orgánica 8/1985, de 3 de julio, reguladora del Derecho a la Educación. (Boletín Oficial del Estado no 159 de 4 de julio de 1985). https://www.boe.es/boe/dias/1985/07/04/ pdfs/A21015-21022.pdf

Ley Orgánica 5/1980, de 19 de junio, por la que se regula el Estatuto de Centros Escolares (Boletín Oficial del Estado no 154 de 27 de junio de 1980). https://www.boe. es/buscar/doc.php?id=BOE-A-1980-13661

Ley 14/1970, de 4 de agosto General de Educación y financiamiento de la reforma educativa, (Boletín Oficial del Estado no 187 de 6 de agosto de 1970). https://www. boe.es/diario_boe/txt.php?id=BOE-A-1970-85212546

López Martín, I. y González Villanueva, P. (2018). La tutoría universitaria como espacio de relación personal. Un estudio de caso múltiple. Revista de Investigación Educativa, 36(2), 381-399. Doi: http://dx.doi.org/10.6018/rie.36.2.291161

McCarthy, S. \& Watson, D. (2018). A New Typology: Four Perspectives of School Counselor Involvement with Families. Journal of School Couseling, 16(1), 1-41.

Martínez-Clares, P., Pérez, J. y Martínez-Juárez, M. (2014). Orientación Profesional en Educación Secundaria. Revista Electrónica Interuniversitaria de Formación del Profesorado, 17(1), 57-71. Doi: http://dx.doi.org/10.6018/reifop.17.1.198841

Martínez-Clares, P. y Martínez-Juárez, M. (2011). La orientación en el S.XXI. Revista Interuniversitaria de Formación del Profesorado (REIFOP), 14(1), 253-265. Doi: http:// dx.doi.org/10.6018/reifop.17.1.198841

Martínez-Otero, V. (2018). Orientación personal y familiar. Madrid: Editorial CCS.

Mekgwe, N. y Kok, A. (2017). The personal career planning needs of secondary school learners in Botswana. International Journal of Educational Sciences, 17(3), 238-246. 10.1080/09751122.2017.1317156

Mendieta Toledo, L. B., Mendieta Toledo, L. R. y Chamba Zambrano, J. M. (2015). Efecto de la aplicación de técnicas de estudio en el rendimiento escolar. In Crescendo. Institucional, Italia, 6(1), 117-137.

Motoso, J. (2002). Innovación psicopedagógica en un contexto escolar público de educación secundaria. En Martínez de la Hidalga, Z. (coord.). Definición y perspectivas profesionales de la Psicopedagogía. (pp.79-95). Bilbao: Universidad de Deusto.

Muelas, A. y Beltrán, J. (2011). Variables influyentes en el rendimiento académico de los estudiantes. Revista de Psicología y Educación, 1(6), 173-195. 
Orden Ministerial del 27 de julio de 2006 por la que se regulan determinados aspectos referidos a la organización y funcionamiento del departamento de orientación en los Institutos de Educación Secundaria. (Boletín Oficial del Estado no 175 de 8 de septiembre de 2006). http://www.juntadeandalucia.es/boja/2006/175/

Otzen, T. y Manterola, C. (2017). Técnicas de muestreo sobre una población a Estudio. International Journal of Morphology, 35(1). Doi: http://dx.doi.org/10.4067/S071795022017000100037

Rodríguez Álvarez, P., Ocampo Gómez, C.I. y Sarmiento Campos, J.A. (2018). Valoración de la orientación profesional en la enseñanza secundaria postobligatoria. Revista de Investigación Educativa, 36(1), 75-91. Doi: http://dx.doi.org/10.6018/rie.36.1.285881

Santana Vega, L. E. y Álvarez Pérez, P. R. (1996). Orientación y educación socio laboral: Una perspectiva curricular. Madrid. EOS.

Silva Teixeira, M. I., Fazendeiro, P.M. \& Braga, A. (2016). A identidade do professor: desafíos colocados pela globalização. Revista Brasileira de Educação, 21(65). Doi: http:// dx.doi.org/10.1590/S1413-24782016216520

Thomas, J. y Nelson, J. (2007). Métodos de investigación en actividad física. Barcelona, España: Paidotribo.

Torrecilla, E., Rodríguez, M.J., Herrera, E. y Martín J. (2013). Evaluación de calidad de un proceso de tutoría de titulación universitaria: la perspectiva del estudiante de nuevo ingreso en educación. Revista Española de Orientación y Psicopedagogía, 24(2), 79-99.

Velaz de Medrano, C., González Benito, A. y López Martín, E. (2018). Assessment of the level of performance of tutoring in secondary education: tutors' own perceptions. Revista de Educación, 382, 107-132.

Velaz de Medrano, C. (2011). La orientación académica y profesional en la Educación Secundaria. En Martín, E.; Solé, I. (Coord.) Orientación Educativa: Modelos y estrategias de intervención. (pp. 129-149). Barcelona: Graó.

Velaz de Medrano, C., Repetto, E., Blanco, A., Guillamon, J.R., Negro, A. y Torrego, J.C. (2001). El desarrollo profesional de los orientadores de Educación Secundaria: Análisis de necesidades y prospectiva. Revista de Investigación Educativa, 19, 199-220.

Villardón, L. y Yáñiz. C. (2013). Propuesta de un Plan de Tutoría y Apoyo a estudiantes de doctorado. Revista de Docencia Universitaria, 11(2), 135-152.

Walker, J. M., Shenker, S. S. \& Hoover-Dempsey, K. V. (2010). Why do parents become involved in their children's education? Implications for school counselors. Professional School Counseling, 14(1), 27-41.

Welsch, D.M. \& Widen, M. (2019). Student gender, counselor gender, and college advice. Education economics, 27(2), 112-131. https://doi.org/10.1080/09645292.2018.1517864

Fecha de recepción: 8 de febrero de 2018 .

Fecha de revisión: 2 de marzo de 2018.

Fecha de aceptación: 26 de marzo de 2019. 